\title{
Exploring Gender Difference in Sleep Quality of Young Adults: Findings from a Large Population Study
}

\author{
Yaqoot Fatima, MTech; Suhail A.R. Doi, MBBS, PhD; Jake M. Najman, PhD; and Abdullah Al Mamun, PhD
}

Objectives: To explore if gender difference in sleep quality is due to higher prevalence of depression in females, and whether socio-demographic and lifestyle factors have a differential effect on sleep quality in males and females.

Methods: Youth self-reports and the Pittsburgh Sleep Quality Index were used to assess sleep quality and associated risk factors. Logistic regression analyses were used to analyze the association between various risk factors and poor sleep quality.

Results: Reports from 3,778 young adults (20.6 \pm 0.86 years) indicate a higher prevalence of poor sleep quality in females than males $(65.1 \%$ vs. $49.8 \%)$. It seems that gender difference in poor sleep is independent of depression, socio-demographics, and lifestyle factors, since the higher odds of poor sleep quality in females was robust to adjust for depression, socio-demographics, and lifestyle factors (OR: I.53, 95\% Cl: I.23-I.90). Lifestyle factors (eg, smoking) (OR I.91; 95\% Cl I.05-3.46) were associated with sleep quality in only males.

Conclusion: Our findings indicate that female vulnerability to poor sleep quality should be explored beyond psycho-social disparities. Perhaps, exploring if the female predisposition to poor sleep quality originates at the biological level could lead to the answer.

Keywords: Young adults; Sleep quality; Gender difference; Depression; Lifestyle

$\mathrm{D}$ ata from recent epidemiological studies provide evidence for the high prevalence of poor sleep quality $(44 \%-60 \%)^{1,2}$ and its impact on cardiovascular problems and other health indicators. ${ }^{3,4}$ Poor sleep quality is often reported with some linked presenting problems and attributed to a range of modifiable, (eg, lifestyle) and nonmodifiable (eg, gender) factors. ${ }^{5}$

Among non-modifiable factors, gender is seen to play a significant role, as many studies report a higher rate of sleep problems in females. ${ }^{6}$ However, the high prevalence of affective disorders in females and other socioeconomic disparities have complicated the role of gender in sleep quality. ${ }^{7,8}$ The association between sleep and affective disorders is well established, and disturbed sleep is considered one of the main symptoms of clinical anxiety and depressive disorders. ${ }^{9}$ Nevertheless, it is unclear if the gender difference in sleep quality can be attributed to higher depression rates in females or other socio-economic disadvantages, or whether it is due to the biological difference in the sleep physiology between males and females. ${ }^{10,11}$

There is a need to ascertain if gender disparity in sleep quality is due to modifiable factors only (eg, depression), so that appropriate intervention can be used to target the underlying cause; or, if the gender difference is arising at biological level, then appropriate prevention measures need to be utilized in predisposed patients. Therefore, further research is needed to examine if the impact of gender on sleep problems is indeed tied to socio-demographic or psychological factors.

Along with socio-demographic and affective disorders, lifestyle has also emerged as a significant predictor of sleep problems and poor sleep quality in young adults. ${ }^{12}$ It is seen that physical inactivity, consumption of alcohol, and long computer screen hours are linked to higher odds of sleep

Received: July 13,2016

Revised: October 15, 2016

Accepted: November I5, 2016

doi: $10.3121 / \mathrm{cmr} .2016 .1338$

Funding Disclosure: A.A.M. is supported by the National Health and Medical Research Council (NHMRC) Career Development Awards (ID 5 I9756). The core study was funded by the National Health and Medical Research Council (NHMRC) of Australia, but the views expressed in the paper are those of the authors and not necessarily those of any funding body. The authors declare no conflict of interests.). 
Table 1. Sleep quality in young adults based on the components of the Pittsburgh Sleep Quality Index (PSQI)*

\begin{tabular}{|c|c|c|c|c|}
\hline \multirow[b]{2}{*}{ PSQI Sleep Quality Components } & \multicolumn{3}{|c|}{$\mathbf{N}(\%)$} & \multirow[b]{2}{*}{$P$ value } \\
\hline & All Subjects & Males & Females & \\
\hline \multicolumn{5}{|l|}{ Component1: Subjective Sleep Quality } \\
\hline Very good/Fairly good & $3,011(80.1)$ & $1,486(83.5)$ & $1,525(77.0)$ & $<0.0001$ \\
\hline Fairly bad/very bad & $750(19.9)$ & $294(16.5)$ & $456(23.0)$ & \\
\hline \multicolumn{5}{|l|}{ Component 2: Sleep Duration } \\
\hline$>7 \mathrm{hrs} /$ night & $2,228(60.3)$ & $1,007(57.6)$ & $1,221(62.8)$ & 0.01 \\
\hline$\leq 7 \mathrm{hrs} /$ night & $1,466(39.7)$ & $742(42.4)$ & 724 ( 37.2$)$ & \\
\hline \multicolumn{5}{|l|}{ Component 3: Sleep Disturbances } \\
\hline \multicolumn{5}{|l|}{ Component 3a: Snoring } \\
\hline$<$ Once/week & $3,106(84.8)$ & 1,369 (79.3) & $1,737(89.6)$ & $<0.0001$ \\
\hline One to $>$ Three times/week & $559(15.2)$ & $358(20.7)$ & $201(10.4)$ & \\
\hline \multicolumn{5}{|l|}{ Component 3b: Waking During Night } \\
\hline$<$ Once/week & $2,110(56.8)$ & $1,190(68.0)$ & $920(46.9)$ & $<0.0001$ \\
\hline One to >Three times/week & $1,602(43.2)$ & $559(32.0)$ & $1,043(53.1)$ & \\
\hline \multicolumn{5}{|l|}{ Component 3c: Restlessness in Sleep } \\
\hline$<$ Once/week & $1,811(48.2)$ & $1,018(58.3)$ & $793(40.2)$ & $<0.0001$ \\
\hline One to >Three times/week & $1,906(51.3)$ & $729(41.7)$ & $1,177(59.8)$ & \\
\hline \multicolumn{5}{|l|}{ Overall Sleep Disturbances $=3 a+3 b+3 c$} \\
\hline$<$ Once/week & $2,296(62.9)$ & $1,205(70.2)$ & $1,091(56.5)$ & $<0.0001$ \\
\hline One to >Three times/week & $1,352(37.1)$ & $512(29.8)$ & $840(43.5)$ & \\
\hline \multicolumn{5}{|l|}{ Component 4: Sleep medication use } \\
\hline Not during the past month & $3,466(92.2)$ & $1,663(93.5)$ & $1,803(91.1)$ & 0.005 \\
\hline$<$ One/week to $>$ Three times/week & $294(7.8)$ & $116(6.5)$ & $178(9.9)$ & \\
\hline \multicolumn{5}{|l|}{ Component 5: Daytime Dysfunction } \\
\hline \multicolumn{5}{|l|}{ Component 5a:Trouble staying awake } \\
\hline Not during the past month & $2,611(69.5)$ & $1,297(72.9)$ & $1,314(66.5)$ & $<0.0001$ \\
\hline$<$ One/week to $>$ Three times/week & $1,146(30.5)$ & $483(27.1)$ & $663(33.5)$ & \\
\hline \multicolumn{5}{|l|}{ Component 5b: Keeping up Enthusiasm } \\
\hline No problem/very slight problem & $3,221(85.6)$ & $1,543(88.4)$ & $1,648(83.2)$ & $<0.0001$ \\
\hline $\begin{array}{l}\text { Somewhat of a problem / Very big } \\
\text { problem }\end{array}$ & $540(14.4)$ & $207(11.63)$ & $333(16.8)$ & \\
\hline \multicolumn{5}{|l|}{ Overall Daytime Dysfunction $=5 a+5 b$} \\
\hline No problem/Very slight problem & $3,436(91.6)$ & $1,671(94.0)$ & $1,765(89.4)$ & $<0.0001$ \\
\hline $\begin{array}{l}\text { Somewhat of a problem / Very big } \\
\text { problem }\end{array}$ & $316(8.4)$ & $107(6.0)$ & $209(10.6)$ & \\
\hline \multicolumn{5}{|l|}{ Overall PSQI* Scored Sleep Quality } \\
\hline Good & $1,500(42.1)$ & $843(50.2)$ & $657(34.9)$ & $<0.0001$ \\
\hline Poor & $2,061 \quad(57.9)$ & $837(49.8)$ & $1,224(65.1)$ & \\
\hline
\end{tabular}

${ }^{*} \mathrm{PSQI}$ : Shortened version of PSQI, doesn't include information on sleep latency and sleep efficiency, $P$ value : Chi-square and Fisher's exact tests 
problems. ${ }^{13}$ However, there is little known about the differential impact of unhealthy lifestyle on sleep quality in male and female young adults. ${ }^{14}$

This study intends to explore if gender difference in sleep quality can be attributed to the higher prevalence of depression in females, and if the socio-demographic and lifestyle factors exhibit gender-specific influence on sleep quality in young adults.

\section{PARTICIPANTS AND METHODS}

This study used the 21 years of follow-up data from the Mater-University of Queensland Study of Pregnancy (MUSP) cohort and its outcomes. The MUSP study is a prospective study of 7,223 women and their offspring who received antenatal care at a major public hospital in South Brisbane between 1981 and 1983 and delivered a live singleton child who was not adopted before leaving the hospital. These mothers and their offspring have been followed-up prospectively with maternal questionnaires being administered when their offspring was 6 months, and 5, 14, and 21 years. At 14 and 21 years, the offspring completed health, welfare, and lifestyle questionnaires. Written informed consent from the mothers was obtained at all data collection phases and from the young adults at the 21-year follow-up of the study. Ethics committees at the Mater Hospital and the University of Queensland approved each phase of the study. Full details of the study participants and measurements are reported elsewhere. ${ }^{15}$ For the 21-year follow-up, young adults completed a comprehensive questionnaire (570 items) related to an array of psycho-socio-demographic attributes, lifestyle and health-related conditions. In this study results are focused on the sleep quality information from the follow-up at age 21 years.

\section{Measures}

\section{Sleep Quality in Young Adults}

In this study, sleep quality was assessed by a shortened version of the Pittsburgh Sleep Quality Index (PSQI). ${ }^{16}$ The PSQI is a comprehensive, self-reported questionnaire that assesses sleep quality and involves seven clinically relevant domains of sleep: subjective sleep quality, sleep latency, sleep duration, habitual sleep efficiency, sleep disturbances, use of sleep medicines, and daytime dysfunction. The components are represented as ordinal variables coded from $0-3$, where 0 indicates "no difficulty" and 3 "severe difficulty." Scores from these separate components are combined to derive a global measure of sleep quality. In this study, we did not have information about sleep efficiency and sleep latency, so the overall sleep quality was assessed using only the remaining five components of the PSQI. Subjective sleep quality was determined by asking how sleep quality had been over the past month. Sleep duration was based on the usual number of hours of sleep per night and then categorized in four groups: sleep duration $>7$ hours (scored 0), 6-7 hours (scored 1), 5-6 hours (scored 2), and $<5$ hours (scored 3). Use of sleep medication was determined by asking about the use in the past month. The sleep disturbance component was based on the reports for waking during the night, snoring, and restlessness in sleep. Daytime dysfunction was determined by asking questions about the ability to stay awake during the daytime and have enthusiasm for usual daytime activities. The scores of different components were summed to yield an overall score (range of 0 to 15 , where 0 indicates no difficulty and 15 indicated severe difficulty). The overall score of $>3$ was considered as an indicator of poor sleep quality.

\section{Socio-Demographic, Behavioral and Psycho-Social Data}

Items from the Young Adult Self Report (YASR) were used to identify socio-demographic, lifestyle, and medical factors for their role in poor sleep quality. ${ }^{17}$ The following variables were used in the analysis: gender, racial origin (White, Asian, Aboriginal-Islander), education (Incomplete secondary, Complete secondary, College/TAFE, and University), marital status (Married/de facto relationship, Single), income levels (using 20\% cutoff: $<\$ 160 /$ week, and $\geq \$ 160 /$ week), and living arrangement (Living with parents/relatives, Independent living). Along with these variables, the following behavioral factors were also used in the analysis: smoking (Non-smoker, $<10$ cigarettes/day, and $\geq 10$ or more cigarettes/day), the frequency and quantity of alcohol consumed (abstainer, light drinker, moderate drinker, heavy drinker, very heavy drinker), illicit drugs intake [eg, cannabis, marijuana, pot] (never used, recreational users including 'once or so' and 'not in the last month', frequent users including 'every day' and 'every few days'), weekday television hours/day (never / $<1$ hours, 1-3 hours, 3-5 hours, $>5$ hours), computer hours/week (None, 1-10 hours, 10-30 hours, $>30$ hours), and the frequency of vigorous exercise (eg, swimming, tennis, netball, athletics, running) for a period of at least 20 minutes (Not at all, 1 or 2 times a week, 3 or more times a week). Depression was assessed using the 20-item version of the Center for Epidemiologic Studies Depression (CES-D) Scale. ${ }^{18}$ The CES-D scale has been constructed using well-known items from existing depression scales, and it measures the severity and persistence of depressive symptoms over a 1-week period. Additionally, tension headaches (Yes, No) and body mass index (BMI) categories (normal BMI 18.5-24.9, overweight BMI 25.0-29.9, obese BMI $\geq 30)^{19}$ were also analyzed for their impact on sleep quality.

\section{Statistical Analyses}

Descriptive statistics, Chi-square, and Fisher's exact tests were done to explore the association of categorical risk factors with sleep quality and the various sleep components of the PSQI. Multivariable logistic regression analysis was used to analyze the association between poor sleep quality and depression as well as socio-demographic, lifestyle, and medical factors. Link tests were used to check for model specification errors, and the C-statistic was computed for each model. All analyses were undertaken using Stata, version 13 (Stata, College Station, TX). 
Table 2. Odds Ratios (OR) for PSQI* assessed poor sleep quality in young adults associated with independent risk factors

\begin{tabular}{|c|c|c|c|c|c|c|}
\hline \multirow[b]{2}{*}{ Risk Factors } & \multicolumn{2}{|c|}{ Model A } & \multicolumn{2}{|c|}{ Model B* } & \multicolumn{2}{|c|}{ Model C* } \\
\hline & OR & $95 \% \mathrm{Cl}$ & OR & $95 \% \mathrm{Cl}$ & OR & $95 \% \mathrm{Cl}$ \\
\hline Female & 1.88 & $1.64-2.15$ & 1.74 & $1.42-2.13$ & 1.53 & $1.23-1.90$ \\
\hline Asian & & & 1.64 & $1.01-2.68$ & 1.80 & $1.07-3.00$ \\
\hline Exercise 3+times/week & & & 0.74 & $0.58-0.95$ & & \\
\hline Smoking $20+$ cigarettes/week & & & 1.73 & $1.09-2.75$ & 1.76 & $1.07-2.88$ \\
\hline Computer use $10-30$ hours/week & & & 1.54 & $1.15-2.05$ & 1.50 & $1.10-2.04$ \\
\hline Frequent use of drugs & & & 2.01 & $1.43-2.83$ & 1.53 & $1.06-2.21$ \\
\hline Occasional use of drugs & & & 1.24 & $1.00-1.55$ & & \\
\hline Tension Headache & & & 2.16 & $1.65-2.82$ & 1.78 & $1.34-2.37$ \\
\hline Depression & & & & & 1.13 & $1.11-1.15$ \\
\hline
\end{tabular}

Risk factors input in the analysis: Model A: Gender (ref: male), Model B: Model A + race (ref: Caucasian), marital status (ref: single: including never married, divorced, widowed, separated), education (ref: incomplete secondary), income (ref: $<\$ 160 /$ week), smoking (ref: Nill), drinking (ref: abstainer), drugs (ref: never used), computer use (ref: none), TV hours (ref: never/<1 h/day), exercise (ref: no), tension headache.

Model C: Model B + depression. *shortened version of PSQI, only significant associations are shown in the table. All models passed the link test, C-statistics model-A: 0.58, Model-B: 0.65, Model-C:0.75

\section{RESULTS}

The results were obtained using reports from a total of 3,778 young adults $(52.6 \%$ female), mean age 20.6 years $(\mathrm{SD}=$ 0.86). The majority of the participants were Caucasian (92.4\%), followed by Aboriginal-Islander (3.9\%), and the remaining were Asian. About half of the study subjects had completed secondary school (52.8\%); there were comparable numbers of subjects who attended TAFE/college or did not complete secondary school, and the remaining subjects $(4.2 \%)$ attended a university. The majority of subjects were living with their parents or relatives $(62.8 \%)$, and a considerably smaller percentage of study subjects were married or in a de facto relationship $(21.1 \%)$, or earning $\geq \$ 160$ per week $(20.6 \%)$.

The results of the PSQI assessed poor sleep quality (Table 1) reveal a significant gender difference in the prevalence of poor sleep quality and sleep problems. Female subjects were found to report a higher prevalence of poor sleep quality $(65.1 \%$ vs. $49.8 \%)$ and all other sleep problems than males, but also reported longer sleep duration than their male counterparts. There was a significant gender difference in the prevalence of poor sleep quality in depressed $(36.3 \%$ males vs. $63.7 \%$ females) as well as non-depressed (42.9\% males, $57.1 \%$ females) subjects. The difference between subjective reports and the PSQI assessed poor sleep quality was overwhelming, as the prevalence of poor sleep quality was only $19.9 \%$ based on self-report (single item), opposed to $57.9 \%$ based on PSQI assessment.

Unadjusted regression analysis indicates that females have higher odds of poor sleep quality than males (OR 1.88; 95\% CI 1.64-2.15) (Table 2). The higher odds of poor sleep in females was still significant, even after adjusting for the role of sociodemographic factors, lifestyle, and medical problems (OR 1.74; 95\% CI 1.42-2.13). In the final model, where we also entered depression in addition to other previuosly mentioned risk factors, female subjects were still found to have higher odds of poor sleep quality (OR 1.53; 95\% CI 1.23-1.90).

We conducted separate regression analysis to explore if there was a difference between males and females for factors affecting sleep quality (Table 3). It was found that racial background (OR 2.20; 95\% CI 1.11-4.36) and lifestyle, such as smoking (OR 1.91; 95\% CI 1.05-3.46), and frequent use of drugs (OR 1.71; 95\% CI 1.09-2.67), were predominantly associated with poor sleep quality in male subjects. Intriguingly, depression had a similar impact on poor sleep quality in males (OR 1.15; 95\% CI 1.12-1.18) and females (OR 1.11; 95\% CI 1.08-1.13), while BMI categories were not seen to be associated with sleep quality in either gender. 
Table 3: Odds Ratios (OR) for PSQ* assessed poor sleep quality in male and female young adults associated with independent risk factors.

\begin{tabular}{|c|c|c|c|c|}
\hline \multirow[b]{2}{*}{ Risk Factors } & \multicolumn{2}{|c|}{ Males } & \multicolumn{2}{|c|}{ Females } \\
\hline & OR & $95 \% \mathrm{Cl}$ & OR & $95 \% \mathrm{Cl}$ \\
\hline \multicolumn{5}{|l|}{ Sociodemographic Factors } \\
\hline Asian & 2.20 & $1.11-4.36$ & & \\
\hline \multicolumn{5}{|l|}{ Lifestyle Factors } \\
\hline Smoking $20+$ cigarettes/week & 1.91 & $1.05-3.46$ & & \\
\hline Frequent use of drugs & 1.71 & $1.09-2.67$ & & \\
\hline \multicolumn{5}{|l|}{ Medical/Psychological Problems } \\
\hline Tension Headache & 1.93 & $1.15-3.23$ & 1.76 & $1.24-2.51$ \\
\hline Depression & 1.15 & $1.12-1.18$ & 1.11 & $1.08-1.13$ \\
\hline
\end{tabular}

Risk factors input in the analysis: race (ref: Caucasian), marital status (ref: single: including never married, divorced, widowed, separated), education (ref: incomplete secondary), income (ref: $<\$ 160 /$ week), smoking (ref: Nill), drinking (ref: abstainer), drugs (ref: never used), computer use (ref: none), TV hours (ref: never/<1 h/day), exercise (ref: no), tension headache, depression. Only significant associations $(P<0.05)$ are shown in the table. All models passed the link test, $C$-statistics- model for males:0.75, model for females:

\section{DISCUSSION}

This study confirms higher prevalence of poor sleep quality in young females. Our findings for the significant gender difference in sleep quality in non-depressed subjects and higher odds of poor sleep quality in females cast doubt on the primacy of depression in leading to the gender difference in sleep quality. Based on the findings of this study, we could argue that though depression is associated with poor sleep, the gender difference in sleep quality appears to be due to reasons other than psychosocial disparities. Additionally, most of the lifestyle factors seem to influence sleep quality in male subjects only; but, being a cross-sectional study, we could not determine the temporal sequence between lifestyle and poor sleep quality. Hence, evidence from longitudinal studies is needed to confirm these associations.

Our results for PSQI assessed sleep quality are in agreement with other studies,,$^{20,21}$ but the self-reported prevalence of poor sleep quality is considerably less than the rate reported in previous studies. ${ }^{22}$ Nonetheless, the perception of sleep quality being a subjective issue is found to be affected by cultural and social practices and racial background; ${ }^{23}$ therefore, the disparity in results may be partly attributed to the difference in study composition. Additionally, in this study, the number of subjects sleeping for short sleep duration was found to be considerably higher than in other studies in Australian adults (39.7\% vs. $16.6 \%) .{ }^{24}$ However, it should be considered that our study had a much more restricted age range than other studies, which might explain the disparity in the results.

The significantly large difference between the self-reported sleep quality and PSQI assessed sleep quality has implications for epidemiological studies relying on just a single question to assess sleep quality. It should be considered that sleep quality is a broad indicator of the adequacy of sleep, and therefore, more correctly assessed by concurrent exploration of the significant domains of sleep, such as initiation, maintenance, duration, perceived adequacy, daytime somnolence, regularity, and the use of sleep medications. ${ }^{25}$ The results of this study suggest that validated sleep questionnaires should be used for assessing subjective aspects like sleep quality in populationbased studies.

Gender difference in poor sleep quality has been previously reported for older populations, ${ }^{26}$ but evidence from some recent studies also found gender difference to be present in sleep quality in young adults. ${ }^{20}$ However, existing studies do not provide information on whether gender difference remains significant after concurrently considering the impact of other socio-demographic, lifestyle factors, and affective disorders. ${ }^{11}$ The gender difference in sleep problems is mainly attributed to the primacy of affective disorders and socioeconomic disparities, suggesting these may be the pathway variables through which gender disparity in poor sleep is exhibited. . $^{5,710}$ In our study, gender difference in sleep quality remained significant even after controlling for sociodemographic and lifestyle factors as well as depression; although, after controlling for these covariates, the effect of gender on sleep quality was slightly attenuated. Therefore, it can be said that the higher prevalence of depression in females does not lead, but rather contributes to the gender difference in poor sleep quality. Female predisposition for poor sleep quality is perhaps driven by the gender-based differences in the biology of sleep ${ }^{27}$ or some other variable we failed to include in our analysis (eg, family history of poor sleep) ${ }^{28}$ and is further aggravated by higher affective disorders in females.

Analyses exploring the differential effect of various risk factors on sleep quality suggest that most of the lifestyle 
factors (eg, smoking, drug abuse) seem to exhibit genderspecific association. Due to the cross-sectional nature of the study, it could not be said whether unhealthy lifestyle preceded sleep quality, or poor sleep quality affected lifestyle. However, considering the study subjects were very young, it may not be unlikely that unhealthy lifestyle preceded poor sleep quality. Similar to lifestyle factors, sociodemographic factors (ie, racial background) have a significant effect on sleep quality on young males, but not females. Our results uphold previous findings of poor sleep in the minority population on sleep problems in young adults, ${ }^{29}$ though evidence from longitudinal studies is needed to explore the differential effect seen in our study. We could not find a role for marital status in sleep quality, but it should be considered that the role of partnership support is mostly explored for older subjects. ${ }^{30}$ Therefore, it would not be appropriate to compare these results with our study, which was based on young adults.

Overall, the results of this study augment the existing evidence for poor sleep in young adults. However, the cross-sectional nature of this study limits capture of long-term trends for sleep quality in the context of aging. Other limitations of this study are use of a shortened version of PSQI and lack of information on anxiety prevalence, which could restrict the accuracy of our results. Moreover, some of the items in PSQI are very "soft" (eg, restless sleep); therefore, our estimates of poor sleep quality prevalence may be higher than the actual prevalence rates. Nonetheless, our results have better reliability and validity than results obtained from studies using only a single question to explore sleep quality.

\section{CONCLUSION}

In conclusion, poor sleep quality is a major problem among young adults, as more than half of the study subjects were found to have poor sleep quality, with even higher prevalence rates for female subjects. It should be noted that gender difference in sleep quality and sleep problems could not be solely attributed to the higher prevalence of affective disorders in females or socio-economic disparities. The differential impact of some lifestyle and sociodemographic factors on poor sleep quality requires further confirmation from longitudinal studies to help in understanding the direction of association and implementation of effective intervention strategies.

\section{ACKNOWLEDGEMENTS}

The authors thank MUSP participants, the MUSP Research Team, the MUSP data collection teams, the Mater Misericordiae Hospital and the Schools of Social Science, Population Health, and Medicine at The University of Queensland for their support; and the National Health and Medical Research Council (NHMRC).

\section{REFERENCES}

1. Kenney SR, LaBrie JW, Hummer JF, Pham AT. Global sleep quality as a moderator of alcohol consumption and consequences in college students. Addict Behav. 2012;37:507 512.

2. Matthews D. Assessing Sleep Quality in Young Adult College Students, Aged 18 - 24 in Relation to Quality of Life and Anthropometrics. The University of Maine; Electronic Theses and Dissertaions. Paper 1265. 2010. Available at: http://digitalcommons.library.umaine.edu/ cgi/viewcontent.cgi?article $=2311$ \& context $=$ etd.

3. Lou P, Zhang P, Zhang L, Chen P, Chang G, Zhang N, Li T, Qiao C. Effects of sleep duration and sleep quality on prevalence of type 2 diabetes mellitus: A 5-year follow-up study in China. Diabetes Res Clin Pract. 2015;109:178-184.

4. Alapin I, Fichten CS, Libman E, Creti L, Bailes S, Wright J. How is good and poor sleep in older adults and college students related to daytime sleepiness, fatigue, and ability to concentrate? J Psychosom Res. 2000;49:381-390.

5. Bruck D, Astbury J. Population study on the predictors of sleeping difficulties in young Australian women. Behav Sleep Med. 2012;10:84-95.

6. Zhang B, Wing YK. Sex differences in insomnia: a metaanalysis. Sleep. 2006;29:85-93.

7. Arber S, Bote M, Meadows R. Gender and socioeconomic patterning of self-reported sleep problems in Britain. Soc Sci Med. 2009;68:281-289.

8. Goldman-Mellor S, Gregory AM, Caspi A, Harrington H, Parsons M, Poulton R, Moffitt TE. Mental health antecedents of early midlife insomnia: evidence from a four-decade longitudinal study. Sleep. 2014;37:17671775 .

9. Benca RM, Obermeyer WH, Thisted RA, Gillin JC. Sleep and psychiatric disorders. A meta-analysis. Arch Gen Psychiatry. 1992;49:651-668; discussion 669-670.

10. Sekine M, Chandola T, Martikainen P, Marmot M, Kagamimori S. Work and family characteristics as determinants of socioeconomic and sex inequalities in sleep: The Japanese Civil Servants Study. Sleep. 2006;29:206-216.

11. Lindberg E, Janson C, Gislason T, Bjornsson E, Hetta J, Boman G. Sleep disturbances in a young adult population: can gender differences be explained by differences in psychological status? Sleep. 1997;20:381-387.

12. Wakasugi M, Kazama JJ, Narita I, Iseki K, Moriyama T, Yamagata K, Fujimoto S, Tsuruya K, Asahi K, Konta T, Kimura K, Kondo M, Kurahashi I, Ohashi Y, Watanabe T. Association between combined lifestyle factors and non-restorative sleep in Japan: a cross-sectional study based on a Japanese health database. PloS one. 2014;9:e108718.

13. Shochat T. Impact of lifestyle and technology developments on sleep. Nat Sci Sleep. 2012;4:19-31. 
14. Heath AC, Eaves LJ, Kirk KM, Martin NG. Effects of lifestyle, personality, symptoms of anxiety and depression, and genetic predisposition on subjective sleep disturbance and sleep pattern. Twin Res. 1998;1:176-188.

15. Najman JM, Bor W, O'Callaghan M, Williams GM, Aird R, Shuttlewood G. Cohort Profile: The MaterUniversity of Queensland Study of Pregnancy (MUSP). Int J Epidemiol. 2005;34:992-997.

16. Buysse DJ, Reynolds CF 3rd, Monk TH, Berman SR, Kupfer DJ. The Pittsburgh Sleep Quality Index: a new instrument for psychiatric practice and research. Psychiatry Res. 1989;28:193-213.

17. Achenbach TM. Manual for the Young Adult Self-Report and Young Adult Behaviour Checklist. Burlington, VT: University of Vermont, Department of Psychiatry: 1997.

18. Radloff LS. The CES-D Scale: A Self-Report Depression Scale for Research in the General Population. Applied Psychological Measurement. 1977;1:385-401.

19. World Health Organization. Obesity: preventing and managing the global epidemic. Report of a WHO Consultation on Obesity. Geneva, Switzerland: WHO; 1997.

20. Hung HC, Yang YC, Ou HY, Wu JS, Lu FH, Chang CJ. The association between self-reported sleep quality and metabolic syndrome. PloS one. 2013;8:e54304.

21. Afandi O, Hawi H, Mohammed L, Salim F, Hameed AK, Shaikh RB, Al Sharbatti S, Khan FA. Sleep Quality Among University Students: Evaluating the Impact of Smoking, Social Media Use, and Energy Drink Consumption on Sleep Quality and Anxiety. Inquiries Journal/Student Pulse. 2013;5:1-3. Available at: http:// www.inquiriesjournal.com/articles/738/sleep-qualityamong-university-students-evaluating-the-impact-ofsmoking-social-media-use-and-energy-drinkconsumption-on-sleep-quality-and-anxiety.

22. Araújo MFM, Lima ACS, Alencar AMPG, Araújo TM, Fragoaso LVC, Damasceno MMC. Sleep quality assessment in college students from Fortaleza-CE. Texto \& Contexto - Enferm [online] 2013;22:352-360. doi:10.1590/S0104-07072013000200011.

23. Song Y, Ancoli-Israel S, Lewis CE, Redline S, Harrison SL, Stone KL. The association of race/ethnicity with objectively measured sleep characteristics in older men. Behav Sleep Med. 2011;10:54-69.

24. Magee CA, Iverson DC, Caputi P. Factors associated with short and long sleep. Prev Med. 2009;49:461-467.

25. Wells GA, Li T, Kirwan JR, Peterson J, Aletaha D, Boers M, Bresnihan B, Dougados M, Idzerda L, Nicklin J, Suarez-Almazor M, Welch V, Tugwell PS. Assessing quality of sleep in patients with rheumatoid arthritis. J Rheumatol. 2009;36:2077-2086.
26. Luo J, Zhu G, Zhao Q, Guo Q, Meng H, Hong Z, Ding D. Prevalence and risk factors of poor sleep quality among Chinese elderly in an urban community: results from the Shanghai aging study. PloS One. 2013;8:e81261.

27. Van Reen E, Sharkey KM, Roane BM, Barker D, Seifer R, Raffray T, Bond TL, Carskadon MA. Sex of college students moderates associations among bedtime, time in bed, and circadian phase angle. J Biol Rhythms. 2013;28:425-431.

28. Sehgal A, Mignot E. Genetics of sleep and sleep disorders. Cell. 2011;146:194-207.

29. Patel NP, Grandner MA, Xie D, Branas CC, Gooneratne N. "Sleep disparity" in the population: poor sleep quality is strongly associated with poverty and ethnicity. BMC Public Health. 2010;10:475.

30. Chen J, Waite L, Lauderdale DS. Partnership Status, Relationship Quality and Sleep among U.S. Older Adults. Population Association of America, 2013 Annual Meeting. New Orleans, LA; 2013. Available at: http://paa2013.princeton.edu/papers/130785.

\section{AUTHOR AFFILIATIONS}

Yaqoot Fatima, MTech*,'; Suhail A.R. Doi, MBBS, PhD ${ }^{*, \xi, \mid}$; Jake M. Najman, PhD*,\#; and Abdullah Al Mamun, $P h D^{*}$

*School of Public Health, University of Queensland, Brisbane, Queensland, Australia

† Mount Isa Centre for Rural and Remote Health, James Cook University, Queensland, Australia

$\neq$ Research School of Population Health, Australian National University, Canberra, ACT, Australia

\$School of Agricultural, Computing, and Environmental Sciences, Universtity of Southern Queensland, Toowoomba, Australia

|College of Medicine, Qatar University, Doha, Qatar

\#School of Social Science, University of Queensland, Brisbane, Queensland, Australia

\section{AUTHOR CONTRIBUTIONS}

Yaqoot Fatima handled the literature review, data analysis and wrote the first draft of the manuscript. Drs. A. A. Mamun and S.A.R. Doi advised on statistical methods and analysis and critically reviewed the draft of the manuscript. Dr. J. M. Najman is responsible for the conceptual development and continued management of the MUSP study and helped in the critical review of the manuscript. All authors contributed to the final version. 